\title{
Bariatrische Operation reduziert mikrovaskuläre Komplikationen bei adipösen Typ-2-Diabetikern
}

\author{
Diabetiker mit Gewichtszunahme erleiden häufiger mikrovaskuläre \\ Komplikationen als solche mit konstantem Gewicht. Die größte \\ Untersuchung zur bariatrisch-chirurgischen Intervention bei diesen \\ Patienten liefert nun differenzierte Resultate.
}

\begin{abstract}
$\mathrm{n}$ der siebenjährigen Beobachtungsstudie befanden sich 4.683 Typ-2-Diabetiker (mittlerer $\mathrm{HbA}_{1 \mathrm{c}} 7,1 \%$ ) mit erheblicher Adipositas (mittlerer BMI 45,2 $\mathrm{kg} / \mathrm{m}^{2}$ ) und einer mittleren Diabetesdauer von 4,5 Jahren im Alter von 20 bis 79 Jahren. Sie unterzogen sich einer Magenbypassoperation (79\%), einer Schlauchmagenoperation (15\%) oder einer Magenbandimplantation (6\%).

Nach einem, drei, fünf und sieben Jahren waren bei $9,5 \%, 29,9 \%, 31,1 \%$ und $40,5 \%$ der Teilnehmer mikrovaskuläre Krankheiten festzustellen. Eine Retinopathie wurde weit häufiger manifest als eine Nephropathie oder Neuropathie. Patienten, bei denen sich eine Remission des Diabetes einstellte $\left(\mathrm{HbA}_{1 \mathrm{c}}<7 \%\right)$, hatten ein um $30 \%$ vermindertes Risiko. Ein Jahr in der Remission reduzierte mikrovaskuläre Komplikationen um durchschnittlich $19 \%$ unabhängig davon, ob sie danach wieder diabetisch manifest $\left(\mathrm{HbA}_{1 \mathrm{c}}>6,5 \%\right)$ wurden; bei einer Remission von fünf Jahren betrug der Effekt $58 \%$.
\end{abstract}

Der wichtigste Risikofaktor für eine mikrovaskuläre Krankheit war das Alter. Die Komplikationsrate war bei 65bis 79-Jährigen 10,5-fach im Vergleich zu 18- bis 29-Jährigen erhöht. Weitere Risikofaktoren waren die Diabetesdauer, eine Insulinbehandlung und ein $\mathrm{HbA}_{1 \mathrm{c}}$ $>6,5 \%$. Der präoperative BMI und das chirurgische Operationsverfahren waren hinsichtlich der Komplikationen ohne Bedeutung.

Coleman DJ et al. Long-term microvascular disease outcomes in patients with type 2 diabetes after bariatric surgery: evidence for the legacy effect of surgery. Diab Care. 2016;39:1400-1407

\section{Kommentar}

Die Besonderheit der vorliegenden Studie besteht darin, dass erstmals eine mikrovaskuläre Risikominderung nicht nur durch eine Remission des Diabetes, sondern auch durch eine Remission mit nachfolgendem „Rückfall", also Rückkehr zur Diabetesmanifestation, eine Risikoreduktion bewirkte. Die Auswirkung war mit 19\% pro Jahr

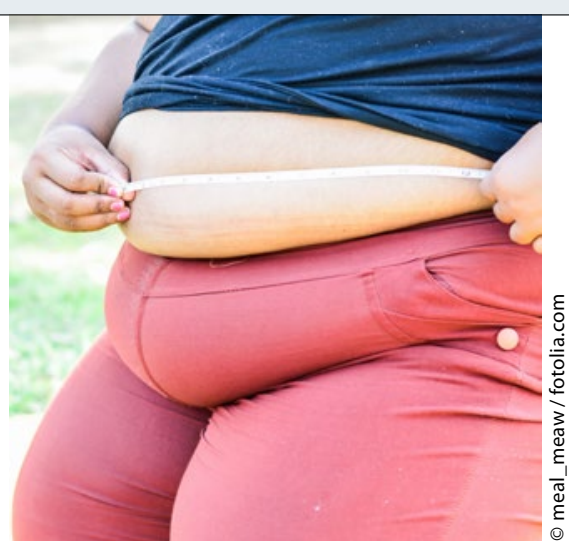

Typ-2-Diabetiker profitieren von einer bariatrischen OP in mehrfacher Hinsicht.

Remission erheblich. Dieser Effekt, metabolisches Gedächtnis oder "legacy effect" genannt, wurde vor Jahren schon bei der Therapie einer Hypercholesterinämie mit Statinen gezeigt.

Fazit: Bei Typ-2-Diabetikern mit einem $\mathrm{HbA}_{1 \mathrm{c}}>6,5 \%$ und einem $\mathrm{BMI}>35 \mathrm{~kg} / \mathrm{m}^{2}$ sollte eine bariatrische Operation grundsätzlich in Erwägung gezogen werden, um die Glykämie zu verbessern, die Adipositas und die damit assoziierten Krankheiten zu mindern, das Sterblichkeitsrisiko zu senken und mikrovaskuläre Krankheiten wie Retinopathie, Nephropathie und Neuropathie zu reduzieren.

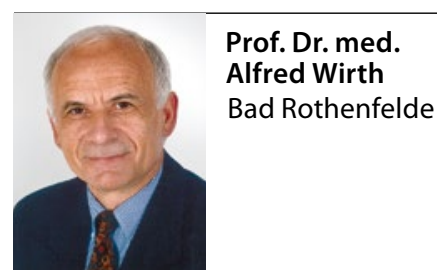

\section{Prognose mit medikamentenbeschichteten Stents nicht besser als mit Metallstents}

Von medikamentenbeschichteten Stents wird eine Reduktion der Restenoserate im Vergleich zu reinen Metallstents erwartet. Welchen Einfluss die Wahl des Stents auf die Prognose hat, wurde in der NORSTENT-Studie untersucht.

$\mathrm{M}$ edikamentenbeschichtete Stents (DES) zur Behandlung einer stenosierenden koronaren Herzerkrankung haben gegenüber reinen Metallstents in einer großen Anzahl randomisierter Studien zu signifikanten Reduktionen der Restenoseraten geführt. Zudem konnten modernere DES Vorteile gegenüber den DES der ersten oder zweiten
Generation zeigen. Darüber hinaus ergaben sich Hinweise darauf, dass möglicherweise die Rate an gefürchteten Stentthrombosen unter Verwendung von DES niedriger sei als bei Verwendung von reinen Metallstents (BMS).

Hieraus erwuchs die Hoffnung, dass DES auch zu einer Reduktion klinisch relevanter kardialer Endpunkte bis hin zu einer Reduktion kardialer Todesfälle führen könnten.

$\mathrm{Ob}$ dies tatsächlich so ist, wurde in der Norwegian Coronary Stent-Studie (NORSTENT) untersucht, die zwischen September 2008 und Mitte Februar 2011 an acht Herzzentren Norwegens durchgeführt wurde. Alle Patienten mit einer signifikanten koronaren Herzerkrankung und der Notwendigkeit einer perkutanen Koronarintervention wurden auf die Verwendung eines DES oder eines BMS randomisiert. Hierzu gehörten über 2.500 Patienten mit stabiler Angina pectoris und über 6.300 Patienten mit akutem Koronarsyndrom. Ausgeschlossen wurden unter anderem Patienten mit einer früheren Koronarintervention. 